Annals of Pure and Applied Mathematics

Vol. 16, No. 2, 2018, 451-460

ISSN: 2279-087X (P), 2279-0888(online)

Published on 27 March 2018

www.researchmathsci.org

Annals of

Pure and Applied

Mathematics

\title{
Unsteady MHD Convective Flow of an Incompressible Viscous Fluid Through Porous Medium Over a Vertical Plate
}

\author{
B.Lakshmanna $^{1}$ and S.Venkateswarl ${ }^{2}$ \\ ${ }^{1}$ Department of Mathematics, Rayalaseema University, Kurnool \\ Andhra Pradesh 518007 Email: laxman7553@gmail.com \\ ${ }^{2}$ Department of Mathematics, RGM College of Engineering and Technology \\ Nandyal, Kurnool, AP, India Email: venkat.6939@gmail.com \\ ${ }^{1}$ Corresponding author
}

Received 28 February 2018; accepted 25 March 2018

Abstract. We have considered the unsteady MHD flow through a loosely packed porous medium in an impulsively started vertical plate with variable heat and mass transfer. The temperature of plate is made to rise linearly with time. The fluid considered is gray, absorbing-emitting radiation but a non-scattering medium. The governing equations involved in the present analysis are solved by the Laplace-transform technique. The velocity, skin friction, Nusselt number and Sherwood number are obtained and computationally discussed for different governing parameters with the combination of the other flow parameters are illustrated graphically, and physical aspects of the problem are discussed.

Keywords: Radiation effects, MHD flows, Heat and mass transfer, vertical plates, porous medium, Skin friction, Nusselt number, Sherwood number.

AMS Mathematics Subject Classification (2010): 05C05, 05C12, 05 C 90

\section{Introduction}

The study of oscillatory flow of an electrically conducting fluid through a porous channel saturated with porous medium is important in many physiological flows and engineering applications such as magneto-hydrodynamic (MHD) generators, arterial blood flow, petroleum engineering and many more. Several authors have studied the flow and heat transfer in oscillatory fluid problems. To mention just a few, Ajibade and Jha [1] presented the effects of suction and injection on hydrodynamics of oscillatory fluid through parallel plates. The same authors extended the problem to heat generating absorbing fluids in [2] while in [3] the effect of viscous dissipation of the free convective flow with time dependent boundary condition was investigated. More recently, Adesanya and Makinde [4] investigated the effect of radiative heat transfer on the pulsatile couple stress fluid flow with time dependent boundary condition on the heated plate. Other interesting cases on hydro magnetic oscillatory fluid flow under different geometries can be found in [5-11] and references therein. Recently, Veerakrishna and Swarnalathamma 


\section{B.Lakshmanna and S.Venkateswarlu}

$[12,13]$ discussed the peristaltic MHD flows. Veerakrishna and Gangadhar Reddy [14,15] discussed MHD free convective rotating flows. Investigation of two-dimensional steady laminar flow with mixed-convection due to a inclined stretching vertical sheet with MHD and partial slip over nanofluidsis done numerically by Pragyal and Vasanthakumari [16]. The combined effects of viscous dissipation and Joule heating on unsteady MHD flow over a stretching sheet saturated in porous medium are analyzed by Sharma and Sharad Sinha [17]. Thirunavukarasu1 and Bhuvaneswari [18] discussed the study of Magnetohydrodynamic flow version of the Casson fluid flow with Hall effect and Rotation in the presence of an inclined magnetic field and porous medium.

In this paper, we have considered radiation effects on MHD flow past an impulsively started vertical plate with variable heat and mass transfer. The results are shown with the help of tables and graphs.

\section{Formulation and solution of the problem}

We consider the flow of unsteady viscous incompressible fluid through a porous medium past a vertical plate. The $x$ - axis is taken along the plate in the upward direction and $y$ axis is taken normal to the plate. Initially the fluid and plate are at the same temperature. A transverse magnetic field $B_{0}$ of uniform strength is applied normal to the plate as shown in figure 1 . The viscous dissipation and induced magnetic field has been neglected due to its small effect. Initially, the fluid and plate are at the same temperature $T_{1}$ and concentration $C_{1}$ in the stationary condition. At time $t>0$, the plate is moving with a velocity $u=u_{0}$ in its own plane and the temperature of the plate is raised to $T_{w}$ and the concentration level near the plate is raised linearly with respect to time.

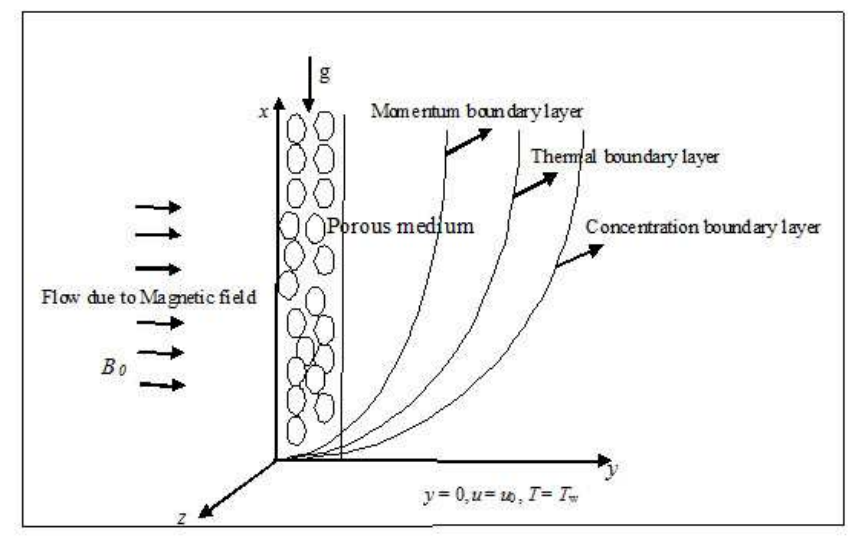

Figure 1: Physical configuration of the problem

The unsteady hydro magnetic equations of the MHD flow through porous medium are as:

$$
\begin{aligned}
& \frac{\partial u}{\partial t}=v \frac{\partial^{2} u}{\partial y^{2}}-\frac{\sigma_{e} B_{0}^{2}}{\rho} u-\frac{v}{K} u+g \beta\left(T-T_{\infty}\right)+g \beta^{*}\left(C-C_{\infty}\right) \\
& \rho C_{p} \frac{\partial T}{\partial t}=k \frac{\partial^{2} T}{\partial y^{2}}-\frac{\partial q_{r}}{\partial y}
\end{aligned}
$$


Unsteady MHD Convective Flow of an Incompressible Viscous Fluid Through Porous Medium Over a Vertical Plate

$\frac{\partial C}{\partial t}=D_{1} \frac{\partial^{2} C}{\partial y^{2}}$

The initial and boundary conditions

$u=0, T=T_{\infty}, C=C_{\infty}, \quad t \leq 0, \forall y$

$u=u_{0}, T=T_{\infty}+\left(T_{w}-T_{\infty}\right) A t, C=C_{\infty}+\left(C_{w}-C_{\infty}\right) A t, \quad y=0$

$u \rightarrow 0, T \rightarrow T_{\infty}, C \rightarrow C_{\infty}, \quad y \rightarrow \infty$

where $A=\frac{u_{0}^{2}}{v}$.

The local radiant for the case of an optically thin gray gas is expressed by

$$
\frac{\partial q_{r}}{\partial y}=-4 a^{*} \sigma\left(T_{\infty}^{4}-T^{4}\right)
$$

Considering the temperature difference within the flow sufficiently small, $T^{4}$ can be expressed as the linear function of temperature. This is accomplished by expanding $T^{4}$ in a Taylor series about $T_{\infty}$ and neglecting higher-order terms, thus

$$
T^{4} \cong 4 T_{\infty}^{3} T-3 T_{\infty}^{4}
$$

Using equations (7) and (8), equation (2) reduces to

$$
\rho C_{p} \frac{\partial T}{\partial t}=k \frac{\partial^{2} T}{\partial y^{2}}+16 a^{*} \sigma\left(T_{\infty}^{3}-T^{4}\right)
$$

Introducing the following non- dimensional quantities:

$$
u^{*}=\frac{u}{u_{0}}, y^{*}=\frac{y u_{0}}{v}, \theta=\frac{T-T_{\infty}}{T_{w}-T_{\infty}}, C^{*}=\frac{C-C_{\infty}}{C_{w}-C_{\infty}}, \mu=\rho v, t^{*}=\frac{t u_{0}^{2}}{v}
$$

Making use of non-dimensional variables, the equations (1), (2) and (9) leads to (dropping asterisks)

$$
\begin{aligned}
& \frac{\partial u}{\partial t}=\frac{\partial^{2} u}{\partial y^{2}}-\left(M^{2}+\frac{1}{D}\right) u+G r \theta+G m C \\
& \frac{\partial \theta}{\partial t}=\frac{1}{P_{r}} \frac{\partial^{2} \theta}{\partial y^{2}}-\frac{R}{P_{r}} \theta \\
& \frac{\partial C}{\partial t}=\frac{1}{S c} \frac{\partial^{2} C}{\partial y^{2}}
\end{aligned}
$$

With boundary conditions

$$
\begin{aligned}
& u=0, \theta=0, C=0, \quad t \leq 0, \quad \forall y \\
& u=1, \theta=t, C=t, \quad y=0 \\
& u \rightarrow 0, \theta \rightarrow 0, C \rightarrow 0, \text { as } y \rightarrow \infty
\end{aligned}
$$




\section{B.Lakshmanna and S.Venkateswarlu}

Where, $R=\frac{16 a^{*} v^{2} \sigma T_{\infty}^{3}}{k u_{0}^{2}}$ is the Radiation parameter, $M^{2}=\frac{\sigma_{e} B_{0}^{2} v}{\rho u_{0}^{2}}$ is the Hartmann number, $D=\frac{k u_{0}^{2}}{v^{2}}$ is the Darcy parameter, $G r=\frac{g \beta v\left(T_{w}-T_{\infty}\right)}{u_{0}^{3}}$ is the thermal Grashof number, $G m=\frac{g \beta^{*} v\left(C_{w}-C_{\infty}\right)}{u_{0}^{3}}$ the mass Grashof number, $\operatorname{Pr}=\frac{\mu C_{p}}{k}$ is Prandtl parameter and $S c=\frac{v}{D_{1}}$ is the Schmidt number.

The dimensionless governing equations (10) to (12), subject to the boundary conditions (13) to (15), are solved by the usual Laplace transform technique. We obtained velocity, temperature, concentration of floe field.

The non-dimensional shear stress is given by

$$
\tau=-\left(\frac{d u}{d y}\right)_{y=0}=-\frac{1}{2 \sqrt{t}}\left(\frac{d u}{d \xi}\right)_{\xi=0}
$$

The non-dimensional Nusselt number is given by

$$
N u=-\left(\frac{d \theta}{d y}\right)_{y=0}=-\frac{1}{2 \sqrt{t}}\left(\frac{d \theta}{d \xi}\right)_{\xi=0}
$$

The non-dimensional Sherwood number is given by

$$
S h=-\left(\frac{d C}{d y}\right)_{y=0}=-\frac{1}{2 \sqrt{t}}\left(\frac{d C}{d \xi}\right)_{\xi=0}
$$

\section{Results and discussions}

Figures (2-8) have been displayed for the velocity, temperature and concentration. Skin friction, Nusselt number and Sherwood number are shown in Tables (1-3). The velocity, temperature and concentration profiles for some realistic values of Prandtl number $\operatorname{Pr}(\operatorname{Pr}$ $=0.71,0.16,3$ for the saturated liquid Freon at $273.3^{\circ}$ and $\operatorname{Pr}=7$ for water) and Schmidt number $\mathrm{Sc}(\mathrm{Sc}=0.2$ for hydrogen) respectively. From figure (2), this presents the velocity profile for different values of $M$ being other parameters fixed. We noticed that the velocity decreases with increasing the Hartmann number $M$. It is due to the fact that the application of transverse magnetic field results a resistive type force (Lorentz force) similar to drag force and upon increasing the intensity of the magnetic field which leads to the deceleration of the flow. Figure (3) is sketched in order to explore the variations of permeability parameter $D$. It is found that the magnitude of the velocity increases with increasing the values of permeability parameter $D$. This is due to the fact that increasing the permeability reduces the drag force which assists the fluid considerably to move fast. Likewise the magnitude of the velocity $u$ reduced continuously with increasing the radiation parameter $\mathrm{R}$ from figure (4). It is clear from figure (5), the velocity decreases with increasing the thermal Grashof number Gr (cooling plate), where as there sharp enhancement in velocity for heating the plate, this is increase sustains away from the 


\section{Unsteady MHD Convective Flow of an Incompressible Viscous Fluid Through Porous Medium Over a Vertical Plate}

plate. The effect of radiation parameter $R$ on the temperature profile is shown in figure (6). It is found that the temperatures, being as decreasing function of $R$, decelerates the fluid flow and reduce the fluid velocity. Such an effect may also be expected, here as increasing radiation parameter $R$ makes the fluid thick and ultimately causes the temperature and thermal boundary layer thickness to reduce. Hence it is observed that the temperature decreases with increasing the radiation parameter $R$ throughout the fluid region. The Prandtl number actually describes the relationship between momentum diffusivity and thermal diffusivity and hence control the relative thickness of the momentum and thermal boundary layers. From Figure (7), we observed that the temperature reduces with increasing the values of Prandtl number Pr, it is also observed that the thermal boundary layer thickness is maximum near the plate and reduces with increasing distances from leading edge and finally approaches to zero. It is also justified due to the fact that thermal conductivity of the fluid decreases with increasing Prandtl number Pr and hence decreases the thermal boundary layer and the temperature profile. Figure (8) depicts the increasing values of Schmidt number $S c$ lead to fall the concentration profiles throughout the fluid.

The numerical values of the skin friction $(\tau)$, Nusselt number $(\mathrm{Nu})$ and Sherwood number $(S h)$ are computed and are tabulated in the tables (1-3), in all these tables the comparison of each parameter is made with first row in the corresponding table. It found from table (1), the effect of each parameter on the skin friction shows that, $\tau$ enhances with increasing $R, D, \operatorname{Pr}, \mathrm{Gr}, \mathrm{Gm}, S c$ and time $t$, while decreases with $M$ and -Gr. It is observed from table (2) that Nusselt number $N u$ increases with increasing $R, \operatorname{Pr}$ and $t$. From table (3) we observed that Sherwood number goes on increasing with increasing $S c$ and $t$.

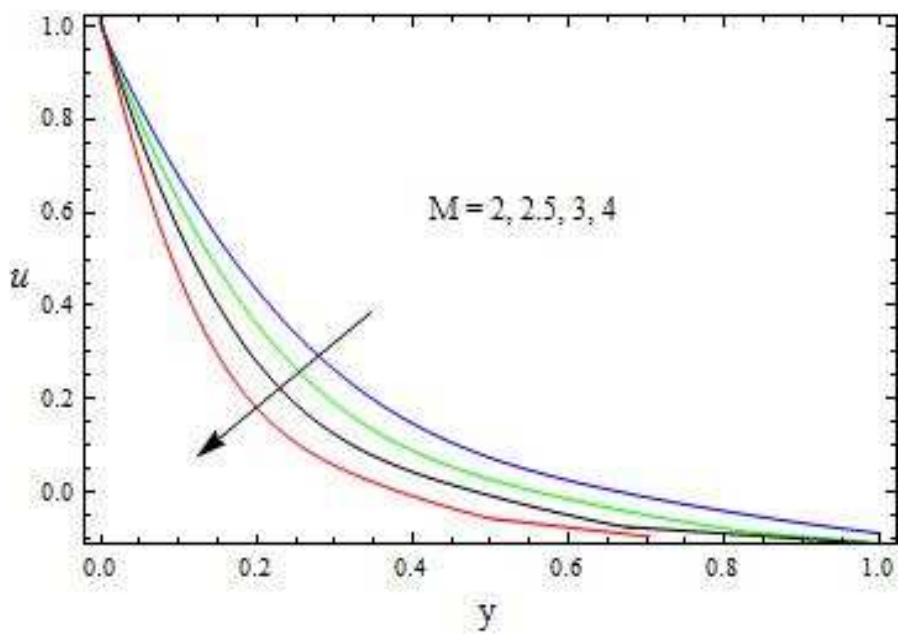

Figure 2: The velocity Profile for $u$ against $M$ with $\mathrm{D}=1 ; \mathrm{P}=0.71 ; \mathrm{t}=0.1 ; \mathrm{Sc}=2 ; \mathrm{R}=1 ; \mathrm{Gr}=5 ; \mathrm{Gm}=10$ 
B.Lakshmanna and S.Venkateswarlu

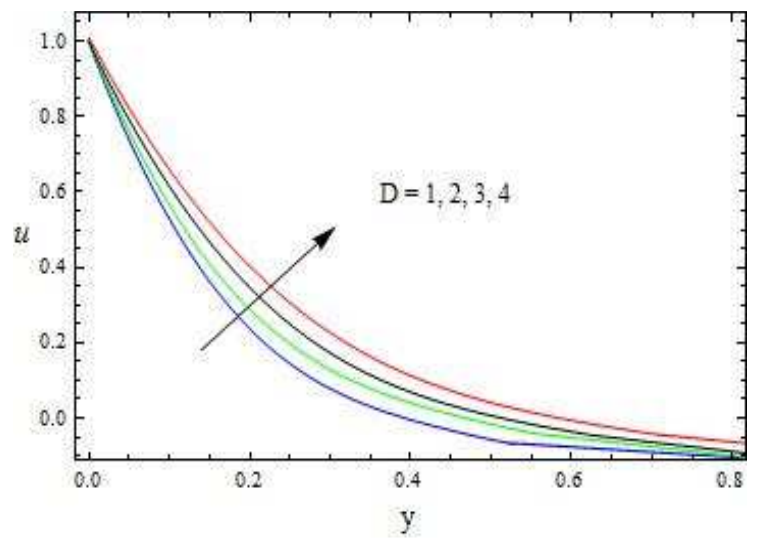

Figure 3: The velocity Profile for $u$ against $D$ with $\mathrm{M}=2 ; \mathrm{P}=0.71 ; \mathrm{t}=0.1 ; \mathrm{Sc}=2 ; \mathrm{R}=1 ; \mathrm{Gr}=5 ; \mathrm{Gm}=10$

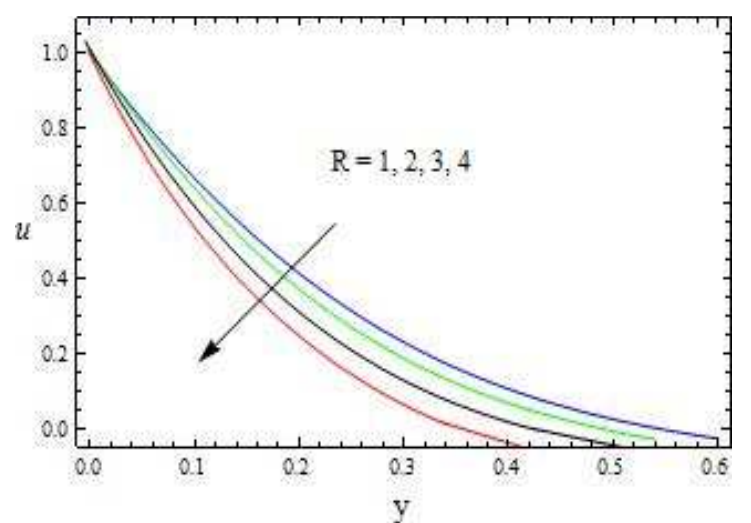

Figure 4: The velocity Profile for $u$ against $R$ with $\mathrm{D}=1 ; \mathrm{P}=0.71 ; \mathrm{t}=0.1 ; \mathrm{Sc}=2 ; \mathrm{M}=2 ; \mathrm{Gr}=5 ; \mathrm{Gm}=1$

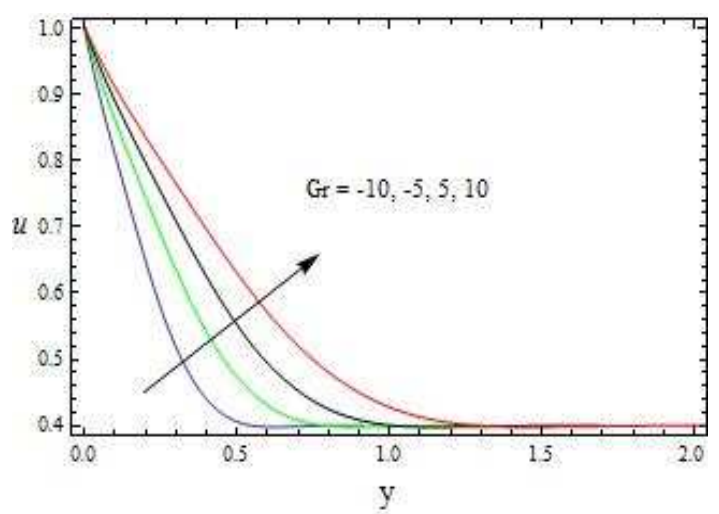

Figure 5: The velocity Profile for $u$ against Gr with $\mathrm{M}=2 ; \mathrm{D}=1 ; \mathrm{P}=0.71, ; \mathrm{Sc}=2 ; \mathrm{R}=1 ; \mathrm{t}=0.1 ; \mathrm{Gm}=10$ 
Unsteady MHD Convective Flow of an Incompressible Viscous Fluid Through Porous Medium Over a Vertical Plate

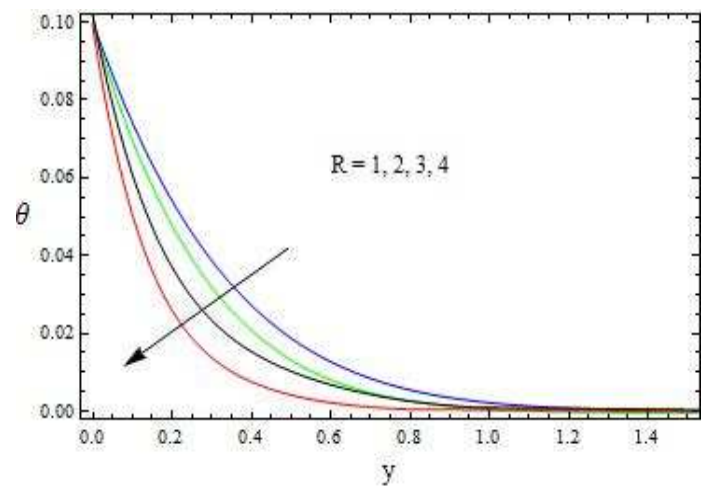

Figure 6: The Temperature Profile for $\theta$ against $\mathrm{R}$ with $\mathrm{P}=0.71 ; \mathrm{t}=0.1$

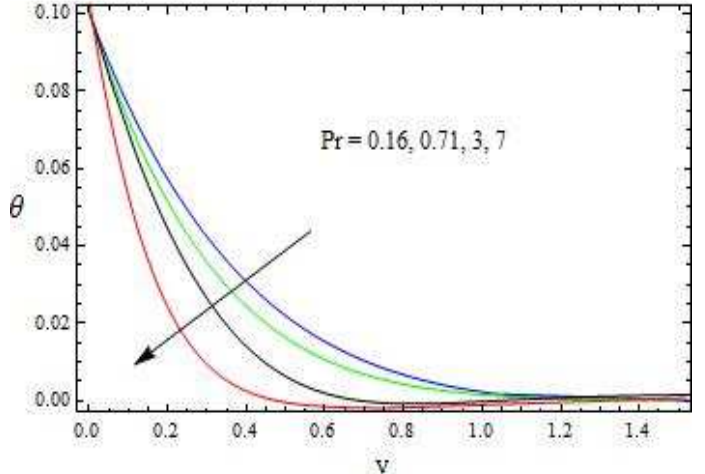

Figure 7: The Temperature Profile for $\theta$ against $\operatorname{Pr}$ with $\mathrm{R}=2$; $\mathrm{t}=0.1$

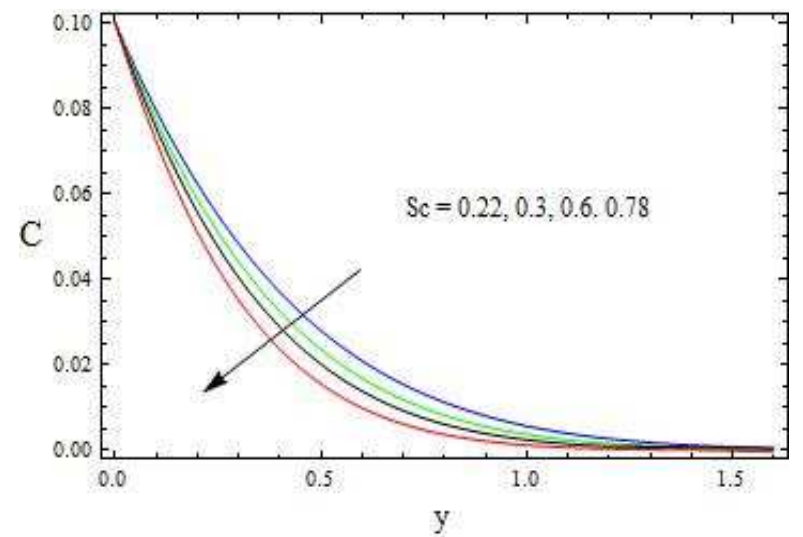

Figure 8: The Concentration Profile for $\mathrm{C}$ against $\mathrm{Sc}$ with $\mathrm{t}=0.1$ 
B.Lakshmanna and S.Venkateswarlu

Table 1: The effects of various parameters on Skin friction (shear stress $(\tau)$ )

\begin{tabular}{|c|c|c|c|c|c|c|c|c|}
\hline $\mathrm{R}$ & $\mathrm{M}$ & $\mathrm{K}$ & $\mathrm{Pr}$ & $\mathrm{Gr}$ & $\mathrm{Gm}$ & $\mathrm{Sc}$ & $\mathrm{t}$ & $\tau$ \\
\hline $\mathbf{1}$ & $\mathbf{2}$ & $\mathbf{1}$ & $\mathbf{0 . 7 1}$ & $\mathbf{5}$ & $\mathbf{1 0}$ & $\mathbf{2}$ & $\mathbf{0 . 2}$ & 3.67042 \\
\hline $\mathbf{2}$ & 2 & 1 & 0.71 & 5 & 10 & 2 & 0.2 & 4.29354 \\
\hline $\mathbf{3}$ & 2 & 1 & 0.71 & 5 & 10 & 2 & 0.2 & 4.84244 \\
\hline 1 & $\mathbf{3}$ & 1 & 0.71 & 5 & 10 & 2 & 0.2 & 3.48338 \\
\hline 1 & $\mathbf{4}$ & 1 & 0.71 & 5 & 10 & 2 & 0.2 & 2.15628 \\
\hline 1 & 2 & $\mathbf{2}$ & 0.71 & 5 & 10 & 2 & 0.2 & 3.70531 \\
\hline 1 & 2 & $\mathbf{3}$ & 0.71 & 5 & 10 & 2 & 0.2 & 3.71552 \\
\hline 1 & 2 & 1 & $\mathbf{0 . 1 6}$ & 5 & 10 & 2 & 0.2 & 3.14544 \\
\hline 1 & 2 & 1 & $\mathbf{3}$ & 5 & 10 & 2 & 0.2 & 5.22357 \\
\hline 1 & 2 & 1 & $\mathbf{7}$ & 5 & 10 & 2 & 0.2 & 10.4094 \\
\hline 1 & 2 & 1 & 0.71 & $\mathbf{1 0}$ & 10 & 2 & 0.2 & 3.92357 \\
\hline 1 & 2 & 1 & 0.71 & $\mathbf{1 5}$ & 10 & 2 & 0.2 & 4.17671 \\
\hline 1 & 2 & 1 & 0.71 & $\mathbf{- 1 0}$ & 10 & 2 & 0.2 & 2.91099 \\
\hline 1 & 2 & 1 & 0.71 & $\mathbf{- 1 5}$ & 10 & 2 & 0.2 & 2.65785 \\
\hline 1 & 2 & 1 & 0.71 & 5 & $\mathbf{5}$ & 2 & 0.2 & 1.96178 \\
\hline 1 & 2 & 1 & 0.71 & 5 & $\mathbf{1 5}$ & 2 & 0.2 & 5.37906 \\
\hline 1 & 2 & 1 & 0.71 & 5 & $\mathbf{2 0}$ & 2 & 0.2 & 7.08770 \\
\hline 1 & 2 & 1 & 0.71 & 5 & 10 & $\mathbf{3}$ & 0.2 & 3.78256 \\
\hline 1 & 2 & 1 & 0.71 & 5 & 10 & $\mathbf{4}$ & 0.2 & 4.36933 \\
\hline 1 & 2 & 1 & 0.71 & 5 & 10 & $\mathbf{5}$ & 0.2 & 4.99703 \\
\hline 1 & 2 & 1 & 0.71 & 5 & 10 & 2 & $\mathbf{0 . 3}$ & 4.84466 \\
\hline 1 & 2 & 1 & 0.71 & 5 & 10 & 2 & $\mathbf{0 . 4}$ & 6.06165 \\
\hline 1 & 2 & 1 & 0.71 & 5 & 10 & 2 & $\mathbf{0 . 5}$ & 7.04960 \\
\hline
\end{tabular}

Table 2: The effects of various parameters on the Rate of heat transfer $(\mathrm{Nu})$

\begin{tabular}{|c|c|c|c|}
\hline $\mathrm{R}$ & $\mathrm{Pr}$ & $\mathrm{t}$ & $\mathrm{Nu}$ \\
\hline $\mathbf{1}$ & $\mathbf{0 . 7 1}$ & $\mathbf{0 . 1}$ & 0.195870 \\
\hline $\mathbf{2}$ & 0.71 & 0.1 & 0.216376 \\
\hline $\mathbf{3}$ & 0.71 & 0.1 & 0.235839 \\
\hline $\mathbf{4}$ & 0.71 & 0.1 & 0.254358 \\
\hline 1 & $\mathbf{0 . 1 6}$ & 0.1 & 0.107555 \\
\hline 1 & $\mathbf{3}$ & 0.1 & 0.634710 \\
\hline 1 & $\mathbf{7}$ & 0.1 & 1.393160 \\
\hline 1 & 0.71 & $\mathbf{0 . 2}$ & 0.331442 \\
\hline 1 & 0.71 & $\mathbf{0 . 3}$ & 0.461249 \\
\hline 1 & 0.71 & $\mathbf{0 . 4}$ & 0.588593 \\
\hline
\end{tabular}


Unsteady MHD Convective Flow of an Incompressible Viscous Fluid Through Porous Medium Over a Vertical Plate

Table 3: The effects of various parameters on the Sherwood number (Sh)

\begin{tabular}{|c|c|c|}
\hline Sc & t & Sh \\
\hline $\mathbf{2}$ & $\mathbf{0 . 1}$ & 0.104512 \\
\hline $\mathbf{3}$ & 0.1 & 0.226218 \\
\hline $\mathbf{4}$ & 0.1 & 0.356825 \\
\hline $\mathbf{5}$ & 0.1 & 0.493120 \\
\hline 2 & $\mathbf{0 . 2}$ & 0.147802 \\
\hline 2 & $\mathbf{0 . 3}$ & 0.181019 \\
\hline 2 & $\mathbf{0 . 4}$ & 0.209023 \\
\hline 2 & $\mathbf{0 . 5}$ & 0.233695 \\
\hline
\end{tabular}

\section{Conclusion}

1. The velocity decreases with increasing the Hartmann number M.

2. The magnitude of the velocity increases with increasing the values of permeability parameter D.

3. The magnitude of the velocity $u$ enhances and reduced continuously with increasing the radiation parameter $\mathrm{R}$.

4. The temperature decreases with increasing the radiation parameter $\mathrm{R}$ or Pr.

5. The increasing values of Schmidt number Sc lead to fall the concentration profiles throughout the fluid.

Acknowledgment. The authors are thankful to all the reviewers for their construction suggestions for improvement of the paper.

\section{REFERENCES}

1. B.K.Jha and A.O.Ajibade, Free convective flow between vertical porous plates with periodic heat input, ZAMM Z. Angew. Math. Mech., (2010) 1-9.

2. B.K.Jha, and A.O.Ajibade, Free convective flow of heat generating absorbing fluid between vertical porous plates with periodic heat input, Int. Commun. Heat Mass Transfer, 36 (2009) 624-631.

3. B.K.Jha and A.O.Ajibade, Effect of viscous dissipation on natural convection flow between vertical parallel plates with timeperiodic boundary conditions, Commun. Nonlinear Sci. Numer. Simul, 17 (2012) 1576-1587.

4. S.O.Adesanya and O.D.Makinde, Heat transfer to magnetohydrodynamic nonNewtonian couple stress pulsatile flow between two parallel porous plates, $Z$. Naturforsch, 67a (2012) 647-656.

5. S.O.Adesanya, E.O.Oluwadare, J.A.Falade and O.D.Makinde, Hydromagnetic natural convection flow between vertical parallel plates with time-periodic boundary conditions, Journal of Magn. Magn. Mater, 396 (2015) 295-303.

6. S.O.Adesanya, Free convective flow of heat generating fluid through a porous vertical channel with velocity slip and temperature jump, Ain Shams Eng. J., 6 (2015) $1045-1052$.

7. S.O.Adesanya, J.A.Falade and O.D.Makinde, Pulsating flow through vertical porous channel with viscous dissipation effect, UPB. Sci. Bull. Ser., D 77 (1) (2015).

8. S.O.Adesanya and J.A.Falade, Thermodynamics analysis of hydromagnetic third grade fluid flow through a channel filled with porous medium, Alexandria Eng. 


\section{B.Lakshmanna and S.Venkateswarlu}

Journal, 54 (2015) 615-622.

9. J.Prakash, B.R.Kumar, R.Sivaraj, Radiation and Dufour effects on unsteady MHD mixed convective flow in an accelerated vertical wavy plate with varying temperature and mass diffusion, Walailak Journal of Science and Technology (WJST), 11 (11) (2014) 939-954.

10. A.Jasmine, Benazir, R.Sivaraj and O.D.Makinde, Unsteady magnetohydrodynamic Casson fluid flow over a vertical cone and flat plate with non-uniform heat source/sink, International Journal of Engineering Research, 21 (2016) 69-83.

11. A.J.Benazir, R.Sivaraj and M.M.Rashidi, Comparison between Casson fluid flow in the presence of heat and mass transfer from a vertical cone and flat plate, Journal of Heat Transfer, 138 (11) (2016) 112005.

12. B.V.Swarnalathamma and M.Veerakrishna, Peristaltic hydrodynamic floe of couple stress fluid through a porous medium under the influence of magnetic field with slip effect, AIP Conf. Proc.1728 (2016) 020603

13. M.Veerakrishna and B.V.Swarnalathamma, Convective heat and mass transfer on MHD peristaltic flow of Williamson fluid with the effect of inclined magnetic field, Conf. Proc., 1728 (2016) 020603.

14. M.Veerakrishna and M.Gangandhar Reddy, MHD free convective rotating flow of visco-elasticfluid past an infinite vertical oscillating porous plate with chemical reaction, IOP Conf. Ser.: Mater. Sci. Eng., 149 (2016) 012217.

15. M.VeraKrishna and G.Subba Reddy, Unsteady MHD convective flow of second grade fluid through a porous medium in a rotating parallel plate channel with temperature dependent source, IOP Conf. Ser Mater. Sci. Eng, 149 (2016) 012216.

16. Pragyal and R.Vasanthakumari, Mixed convective nanofluid flow over an inclined stretching plate with MHD and effects of suction and internal heat generation, Annals of Pure and Applied Mathematics, 12(1) 2016 69-83.

17. P.R.Sharma and Sharad Sinha, Combined effects of viscuss dissipation and joule heating on unsteady MHD flow and Heat transfer over a stretching sheet saturated in porous medium, Annals of Pure and Applied Mathematics, 14(3) (2017) 387-399.

Appendix:

$$
\begin{aligned}
& \xi=\frac{y}{2 \sqrt{t}}, a_{1}=1+\frac{\xi P r}{\sqrt{R t}}, a_{2}=1-\frac{\xi P r}{\sqrt{R t}} a_{3}=\frac{R-\left(M^{2}+\frac{1}{D}\right)}{1-\operatorname{Pr}} a_{4}=\frac{\left(M^{2}+\frac{1}{D}\right)}{S c-1} \\
& a_{5}=\frac{1}{2}\left(a_{9}+a_{10}\left(t-\frac{y}{2 \sqrt{M^{2}+(1 / D)}}\right)\right), a_{6}=\frac{1}{2}\left(a_{9}+a_{10}\left(t+\frac{y}{2 \sqrt{M^{2}+(1 / D)}}\right)\right) \\
& a_{7}=\frac{a_{11}}{2}\left(1+a_{3} t-\frac{y a_{3} \sqrt{\operatorname{Pr}}}{2 \sqrt{R / \operatorname{Pr}}}\right), \quad a_{8}=\frac{a_{11}}{2}\left(1+a_{3} t+\frac{y a_{3} \sqrt{\operatorname{Pr}}}{2 \sqrt{R / \operatorname{Pr}}}\right), a_{9}=1+a_{11}+a_{12}, \\
& a_{10}=\frac{G r}{a_{3}(1-\operatorname{Pr})}+\frac{G m}{a_{4}(1-S c)}, a_{11}=\frac{G r}{a_{3}^{2}(1-\operatorname{Pr})}, \quad a_{12}=\frac{G m}{a_{4}^{2}(1-S c)}
\end{aligned}
$$

